Onkologe 2021 $27: 192-194$

https://doi.org/10.1007/s00761-020-00896-2

Angenommen: 21. Dezember 2020

(c) Springer Medizin Verlag GmbH, ein Teil von Springer Nature 2021

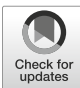

\author{
M. Ghadimi' · A. Reinacher-Schick ${ }^{2}$ H. Bläker ${ }^{3} \cdot$ K. Höffken ${ }^{4}$ \\ ${ }^{1}$ Klinik für Allgemein-, Viszeral- und Kinderchirurgie, Universitätsmedizin Göttingen, Göttingen, \\ Deutschland \\ ${ }^{2}$ Katholisches Klinikum, Ruhr-Universität Bochum, Bochum, Deutschland \\ ${ }^{3}$ Institut für Pathologie, Universitätsklinikum Leipzig, Leipzig, Deutschland \\ ${ }^{4}$ Berlin, Deutschland
}

\title{
State of the Art in der Therapie des Kolonkarzinoms
}

höriger beeinflusst, beschreiben $S$. Aretz und Mitautoren in ihrem Übersichtsartikel.

Der absolute Goldstandard der Diagnostik ist nach wie vor die Koloskopie. Verbesserungen der endoskopischen Technik ermöglichen hierbei nicht nur eine immer frühere und sichere Erkennung von kleinen Adenomen und Karzinomvorstufen, sondern ermöglichen auch im Falle von größeren Adenomen und Frühkarzinomen eine endoskopische Resektion. A. König und Mitautoren geben eine Übersicht, was die Endoskopie heute in der Lage ist zu leisten.

Der histomorphologischen Unterscheidung zwischen Vorstufen und malignen Befunden kommt eine enorme Bedeutung zu. Bereits in der ersten bioptischen Sicherung der Diagnose können Aussagen über genetische Marker und Wachstumsverhalten wie zum Beispiel Tumor-Budding Entscheidungshilfen zur Planung der weiteren Therapie liefern. H. Bläker skizziert die aktuellen histomorphologischen Kriterien und molekularpathologische Befunde und deren Relevanz in der Therapieplanung.

Neben der molekularpathologischen Tumormorphologie sind die Tumorlokalisation und Tumorausdehnung entscheidend für die Therapieplanung. Die chirurgische Entfernung des tumortragenden Kolonabschnitts mit systematischer Lymphadenektomie in offener oder minimal-invasiver Technik stellt die einzige kurative Therapieoption dar. M. von Heesen und $M$. Ghadimi diskutieren detailliert in ihrem Beitrag die Vor- und Nach- teile minimal-invasiver chirurgischer Resektionsverfahren.

Das Wachstumsverhalten eines Kolonkarzinoms ist extrem variabel. Die Spanne reicht von langsam wachsenden Karzinomen, die weder andere Organe infiltrieren noch metastasieren, bis $\mathrm{zu}$ hochmalignen Wachstumsformen, die rasch metastasieren und häufig und rasch rezidivieren. Durch modifizierte adjuvante Therapien kann bei Patienten mit lokal fortgeschrittenen oder nodal positiven Tumoren das tumorfreie Überleben deutlich verlängert werden. Welche Patienten von welcher Therapie profitieren und mit welchen Risiken und Nebenwirkungen die Therapien verbunden sind, beschreiben A. ReinacherSchick und Mitautoren in ihrem Beitrag.

Metastasierte Erkrankungsverläufe sind nur noch in seltenen Fällen heilbar. Dennoch kann auch hier durch multimodale und sog. personalisierte (molekular basierte) Therapiekonzepte häufig eine Langzeitüberleben und in Einzelfällen auch eine Heilung erreicht werden. Welche Kombinationstherapien zur Verfügung stehen, erörtern A. Kurreck und das Team um S. Stintzing.

Durch die zunehmende Komplexität der Behandlung liegt der Gedanke nahe, die Therapie eines Kolonkarzinoms nur noch in zertifizierten Darmkrebszentren anzubieten. $\mathrm{Ob}$ ein solches Vorgehen die Versorgungsqualität entscheidend verbessern kann, diskutieren J. Rückher, S. Wesselmann u. T. Seufferlein.

Schriftleiter und Autoren wünschen den Lesern eine angenehme und erkenntnisreiche Lektüre dieses Schwerpunkt- 
Hier steht eine Anzeige.

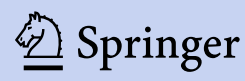




\section{Einführung zum Thema}

hefts. Es wird Ihnen einen aktuellen und umfassenden Überblick über die aktuelle Diagnostik und Therapie sowie Versorgungsstrukturen des Kolonkarzinoms in Deutschland geben.

Michael Ghadimi

Für die Schriftleiter

Klaus Höffken

Für die Herausgeber

\section{Korrespondenzadresse}

Prof. Dr. M. Ghadimi
Klinik für Allgemein-,
Viszeral- und Kinderchirurgie,
Universitätsmedizin
Göttingen
Robert-Koch-Str. 40,
37075 Göttingen,
Deutschland
mghadim@
uni-goettingen.de

Interessenkonflikt. M. Ghadimi, A. Reinacher-Schick, H. Bläker und K. Höffken geben an, dass kein Interessenkonflikt besteht.

Hier steht eine Anzeige.

\section{Springer}

